\title{
A compact very wideband amplifying filter based on RTD loaded composite right/left-handed transmission lines
}

\author{
Mahmoud O. Mahmoud Abu-marasa and Hala Jarallah El-Khozondar ${ }^{*}$
}

*Correspondence: hkhozondar@iugaza.edu Electrical Engineering Department, Islamic University of Gaza, Gaza, Palestine

\begin{abstract}
The composite right/left-handed (CRLH) transmission line $(T L)$ is presented as a general TL possessing both left-handed (LH) and right-handed (RH) natures. $\mathrm{RH}$ materials have both positive permittivity and positive permeability, and LH materials have both negative permittivity and negative permeability. This paper aims to design and analyze nonlinear CRLH-TL transmission line loaded with resonant tunneling diode (RTD). The main application of this design is a very wideband and compact filter that amplifies the travelling signal. We used OrCAD and ADS software to analyze the proposed circuit. CRLH-TL consists of a microstrip line which is loaded with complementary split-rings resonators (CSRRs), series gaps, and shunt inductor connected parallel to the RTD. The designed structure possess a wide band that ranges from 5 to $10.5 \mathrm{GHz}$ and amplifies signal up to $50 \%$. The proposed design is of interest to microwave compact component designers.
\end{abstract}

Keywords: CRLH-TL, Left-handed materials, RTD, Filter, Amplifier, SRRs

\section{Background}

The resonant tunneling diodes (RTD) that pocesses negative differential resistance region have attracted a lot of attention. RTD has high switching speed up to $2.2 \mathrm{THz}$ (Ling 2014). It has been shown that it is possible to monolithically integrate RTD with an optical waveguide electroabsorption modulator (Figueiredo et al. 2001), with laser diode (Slight et al. 2008) and with transmission line analog to digital converter (El-Khozondar et al. 2013a) or signal reshaping (El-Khozondar et al. 2013b).

In 2004, Falcone et al. (2004a) synthesized the first MTMs from CSRRs. Later, the first LH line based on CSRRs was achieved by Falcone et al. (2004b). In a study performed in 2007 (Gil et al. 2007), researchers used LH/RH transmission lines based on CSRRs to achieve a compact very wideband filter ranges between 5-10 GHz. The TL consists of three units.

In a recent work by Maezawa et al. (2010), an amplified signal is achieved when a signal travels through CRLH transmission line loaded with RTD pairs. Authors achieved wide frequency range between 100 and $200 \mathrm{GHz}$. Their TL consists of 160 units.

The main goal of this paper is to study left- and right-handed transmission line based on CSRRs loaded with RTD to obtain ultra-wideband (UWB) and filter with amplified

(c) 2015 Abu-marasa and El-Khozondar. This article is distributed under the terms of the Creative Commons Attribution 4.0 International License (http://creativecommons.org/licenses/by/4.0/), which permits unrestricted use, distribution, and reproduction in any medium, provided you give appropriate credit to the original author(s) and the source, provide a link to the Creative Commons license, and indicate if changes were made. 
output signal. Our model is a modification to the hybrid model (Gil et al. 2007) by loading TL with RTD. The hybrid model is a CRLH-TL composed of a microstrip line loaded with complementary split-rings resonators (CSRRs), series gaps, and shunt inductor (Gil et al. 2007). In our work, we are looking to obtain a compact ultra-wideband filter that modifies the travelling signal. In Section II, the I-V characteristic of the RTD is presented. A prototype of the device is introduced in section III. Conclusion is given in section IV.

\section{Resonant tunneling diode}

Resonant tunneling diode (RTD) has unique applications due to the high switching speed and its negative differential resistance. Figure 1 shows RTD I-V characteristic curve.

The characteristic RTD curve presented in Fig. 1 show the nonlinear behavior of the RTD. There are three regions: the circled one is the region with negative resistance while the regions below and above the negative resistance region has positive resistance value. The characteristic curve shown in Fig. 1 is formulated by the following expression (Reddy 1997).

$$
f(V)=A \ln (\alpha)+H\left(e^{n_{2} q V(t) / K_{B} T}-1\right)
$$

where $\alpha=\left[\frac{1+e^{q\left(B-C+n_{1} V(t)\right) / K_{B} T}}{1+e^{q\left(B-C-n_{1} V(t)\right) / K_{B} T}}\right]\left[\frac{\pi}{2}+\tan ^{-1}\left(\frac{C-n_{1} V(t)}{D}\right)\right], f(V)$ is the current density as function of $V$ which is the voltage cross RTD, $K_{B}$, T, and $q$ are Boltzmann constant, temperature in Kelvin and electron charge in coulombs, respectively. $A, B, C$ and $D$ are the dependent parameters on the device's physics. The experimental RTD I-V characteristic is fitted using the physics based description of the RTD equation in Fig. 1. The fitting parameters are taken from (Slight et al. 2008) as follows, $A=6.48 \times 10^{-3}, B=0.0875$, $C=0.1449, D=0.02132, H=7.901 \times 10^{-4}, n_{1}=0.1902, n_{2}=0.0284$, and $T=300 \mathrm{~K}$.

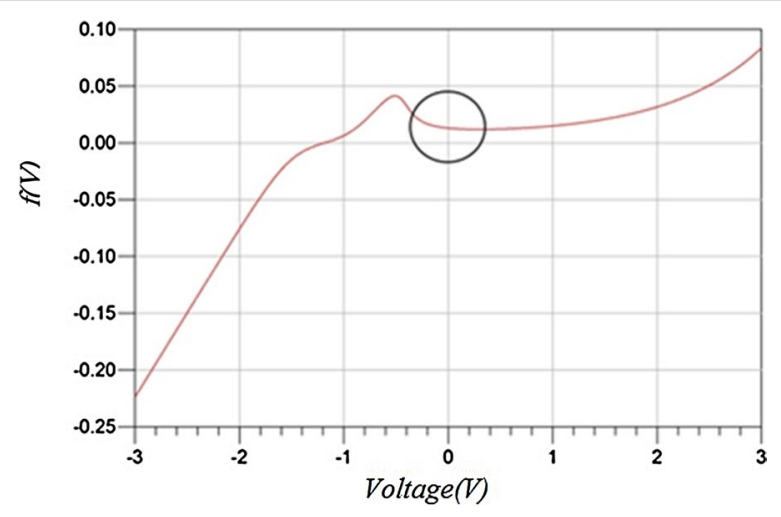

Fig. 1 I-V characteristics of RTD. The circle marks the negative differential resistance (NDR) region 


\section{The proposed filter-amplifier}

The proposed filter-amplifier is constructed by combining the RTD with CRLH-TL based on hybrid CSRR (Gil et al. 2007). The filter introduced in (Gil et al. 2007) consists of four cells with active area below $1 \mathrm{~cm}^{2}$, bandwidth that fluctuates from 5 to $10 \mathrm{GHz}$ and ripple below $1 \mathrm{~dB}$. Figure 2 presents the unit cell and its equivalent lumped circuit. The unit cell is designed with the series resonance frequency as close as possible to the higher resonance of the shunt impedance $\left(\omega_{\mathrm{S}}=\omega_{\mathrm{PH}}\right)$.

We design our filter-amplifier by adding RTD parallel to $\mathrm{L}_{\mathrm{d}}$ as illustrated in Fig. 3. The parameters are (Gil et al. 2007): $\mathrm{L}=2.6 \mathrm{nH}, \mathrm{Cg}=0.2 \mathrm{pF}, \mathrm{L}_{\mathrm{d}}=5.4 \mathrm{nH}, \mathrm{C}_{1}=305 \mathrm{pF}$, $\mathrm{C}_{\mathrm{C} 1}=0.65 \mathrm{pF}, \mathrm{L}_{\mathrm{C} 1}=0.55 \mathrm{nH}, \mathrm{C}_{2}=0.21 \mathrm{pF}, \mathrm{C}_{\mathrm{C} 2}=0.32 \mathrm{pF}$, and $\mathrm{L}_{\mathrm{C} 2}=0.27 \mathrm{nH}$. The RTD was biased in the NDR region using a series variable DC voltage supply. Figure 4 demonstrates the simulated reflection and transmission coefficient of a single cell with RTD, corresponding to the filter (Fig. 3) using Agilent ADS program. Moreover, it can be seen from Fig. 4 that the effect of RTD on transmission coefficient $\left(\mathrm{S}_{21}\right)$ goes above $0 \mathrm{~dB}$. This means that the output power of the filter is bigger than the input power.

The proposed filter allows a signal to pass in wide range starting at $5 \mathrm{GHz}$ and ends at 10.5 GHz. In addition, this filter is capable to amplify the signal.

We simulated the value of the output signal and compared it with the value of the input signal to understand the amplification behavior of our filter. Figure 5 presents the transient response of the input and output signals of the designed filter (Fig. 3) at $9 \mathrm{GHz}$

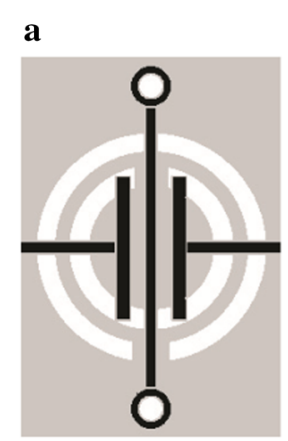

b

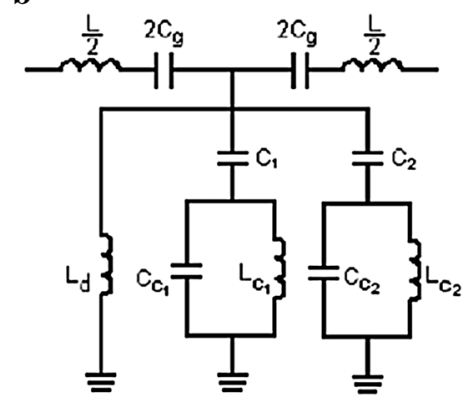

Fig. 2 a The structure of the hybrid left-handed cell. b Equivalent circuit of one hybrid left-handed cells

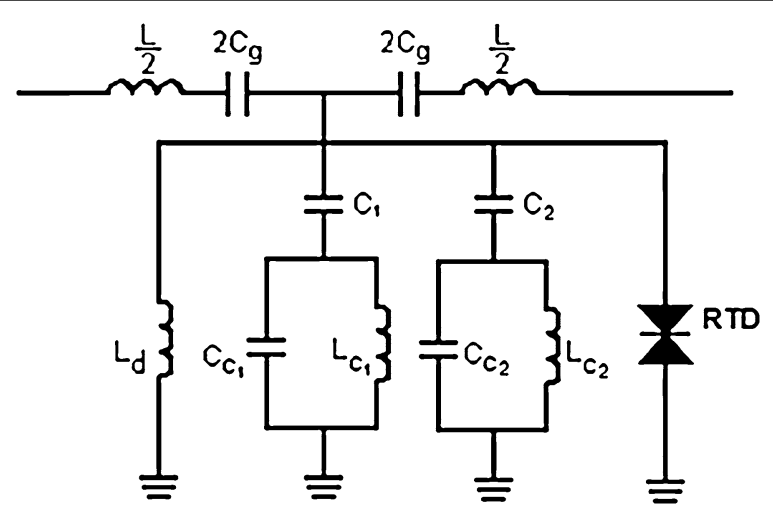

Fig. 3 Equivalent circuit model of the unit cell (Fig. 2) loaded with RTD 


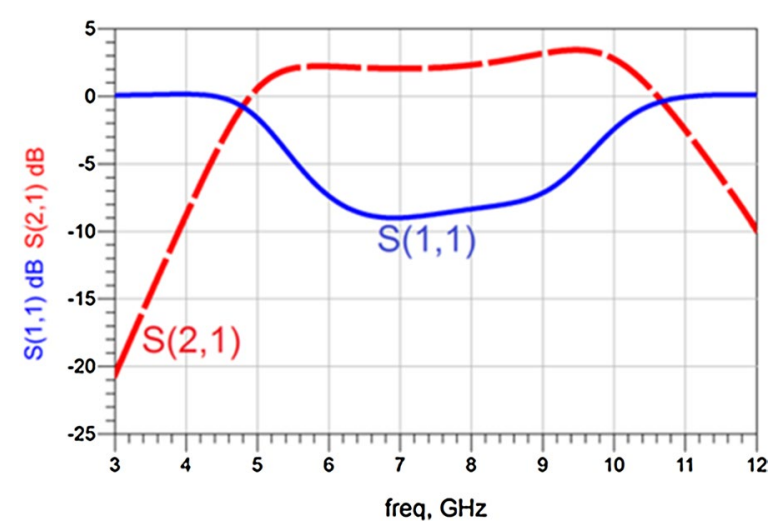

Fig. 4 Simulated reflection and transmission coefficients of a single cell loaded with RTD

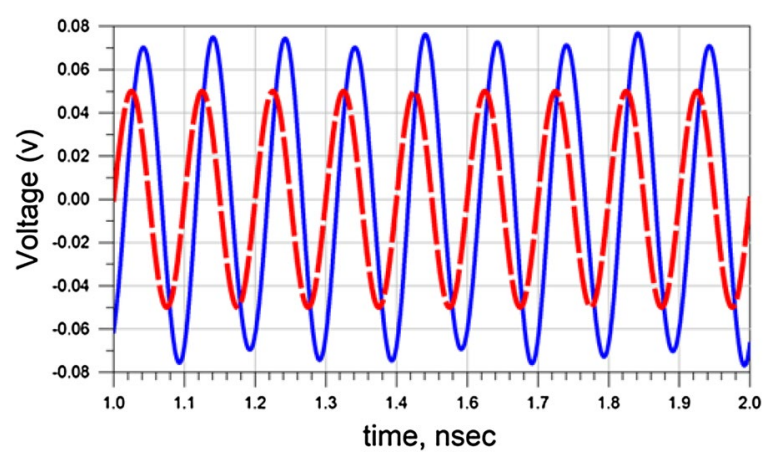

Fig. 5 Simulation result to the equivalent circuit shown in Fig. 3

when DC biasing is set to be $1.18 \mathrm{~V}$. The dashed line refers to the input signal and the sold line refers to the output signal of the filter. The amplification of the input signal is clearly observed. The peak value of the input value is $50 \mathrm{mV}$ while the amplified output signal has a peak value equal to $75 \mathrm{mV}$.

We have noticed that the amplification is $50 \%$. Further, we realized that we could not excite our filter with input more than $300 \mathrm{mV}$, because above this voltage the filter changes the shape of the input signal.

To understand the effect of RTD, we have focused on the negative resistance region (RD). From Fig. 1, we noticed that RD region ranges from -0.1 to $0.1 \mathrm{~V}$. Additionally, the relationship between voltage and currant in the RD region is almost linear. As a result, we can replace the original equation of RTD by approximated Eq. (2):

$$
I=(\text { slope }) V=A^{\prime} V
$$

where $A^{\prime}$ is the slop of currant at the working regain and $V$ is the voltage drop at RTD terminal. The slop is approximately equal to 0.09 . To examine the approximated equation of RTD, we have replaced the RTD in Fig. 3 by a variable current source which depends on the voltage across the terminals of RTD such as $\mathrm{I}=0.09 \mathrm{~V}$.

Figure 6, displays the simulated results when RTD is replaced with current source. The result is similar to the results shown in Fig. 5 . Meaning that the output signal is amplified compared to the input signal. 


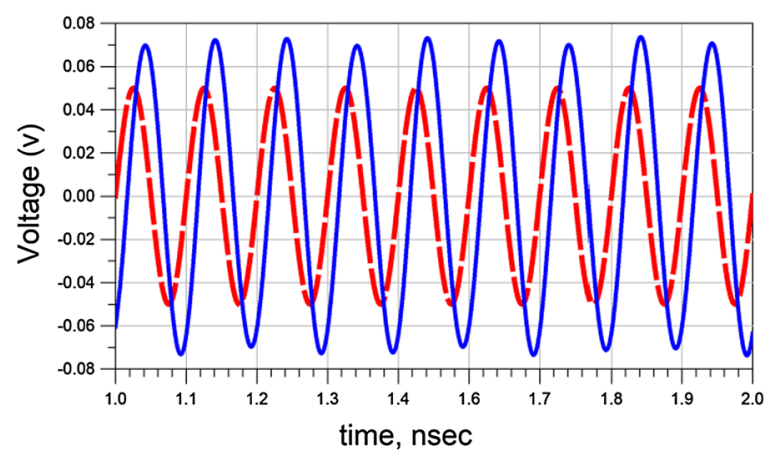

Fig. 6 Reports the simulation result of the equivalent circuit with approximated RTD (Eq. 2) at $9 \mathrm{GHz}$. The dash line refers to the input signal and the sold line refers to the output signal of the filter

Thus, we conclude that for our calculations, the simplified model [Eq. (2)] of RTD can replace the complex representation of RTD characteristic that appears in Eq. (1).

\title{
Conclusion
}

We have designed a compact UWB filter-amplifier by adding RTD to CRLH-TL hybrid unit cell. The bandwidth ranges from 5 to $10.5 \mathrm{GHz}$ and the output signal is amplified compared to the input signal. This is a unique presentation of a filter-amplify based on CRLH-TL loaded with RTD. The designed filter is useful to get rid of the interfering signals below the frequency region of interest. It also can amplify the signal at voltages below $0.3 \mathrm{~V}$. Thus, this filter is good in antenna applications.

\begin{abstract}
Authors' information
Mahmoud O. Mahmoud Abu-Marasa received the Master Degree of the Communication Engineering from Islamic University of Gaza in 2014. He earned his BSc of the Communication and Control Engineering from Islamic University of Gaza in 2005. His research interest includes Nonlinear Transmission Line and Metamaterials. He contributed in local and international conferences. Hala J. El-Khozondar is a professor at the electrical engineering department in the Islamic University of Gaza, Palestinian Territory. She had a Postdoc award at Max Planck Institute in Heidelberg, Germany in 1999. She is a fellow for the world academy of science (FTWAS) and for the Arab region FTWAS-ARO. She is also nominated by Technical University of Munich to become alumni ambassador for research. She is in the editorial board for national and international journals. She worked in initiating and developing the quality assurance unit and the external relations at Islamic University of Gaza. Her research covers a broad spectrum of research includig optical fibres, wireless communication, optical communication, nonlinear optics, optical fiber sensors, magneto-optical isolators, optical filter, MTMs devices, biophysics, electro-optical waveguides, and numerical simulation of microstructural evolution of polycrystalline materials. She has several publications in highly ranked journals. She is a recipient of international awards and recognitions, including a Fulbright Scholarship, DAAD short study visit, a Alexander von Humboldt-Stiftung Scholarship, Erasmus Mundus, the Islamic University Deanery Prize for applied sciences, ICESCO prize and TWAS prize medal. She is also coordinator for several projects including TEMPUS for promoting long life learning in Palestine, and Al-maqdisi to enhance collaboration with French partners.
\end{abstract}

\section{Authors' contributions}

HK were involved in formulating the problem idea, study design and manuscript preparation. MM were involved in the graphical presentation using suitable software and in preparing the manuscript. All authors read and approved the final manuscript.

\section{Competing interests}

The authors declare that they have no competing interests.

Received: 18 December 2014 Accepted: 11 November 2015

Published online: 25 November 2015

\section{References}

El-Khozondar HJ, El-Khozondra RJ, AL-Farra A, Essimbi BZ (2013a) Nonlinear resonant tunnelling diode (RTD) circuits for microwave A/D conversion. Optik 124:6100-6103 
El-Khozondar HJ, El-Khozondra RJ, Essimbi BZ (2013b) Pspice modeling of nonlinear transmission lines analog to digital conversion. Rev Appl Phys (RAP) 2(1):12-16

Falcone F, Lopetegi T, Baena JD, Marqués R, Martín F, Sorolla M (2004a) Effective negative- $\varepsilon$ stop-band microstrip lines on complementary split ring resonators. IEEE Microw Wireless Compon Lett 14:210-282

Falcone F, Lopetegi T, Lao MAG, Baena JD, Bonache J, Marqués R, Martín F, Sorolla M (2004b) Babinet principle applied to the design and metasurfaces and metamaterials. Phys Rev Lett 93:197401-197404

Figueiredo JML, Ironside CN, Stanley CR (2001) Electric field switching in a resonant tunneling diode electroabsorption modulator. IEEE J Quantum Electron 37:1547-1552

Gil M, Bonache J, Garcia-Garcia J, Martel J, Martin F (2007) Composite right/left-handed metamaterial transmission lines based on complementary split-rings resonators and their applications to very wideband and compact filter design. IEEE Trans Microw Theory Tech 55:1296-1304

Ling J (2014) Resonant tunneling diodes: Theory of operation and applications, University of Rochester, Rochester, NY. http://www.ece.rochester.edu/courses/ECE423/ECE223_423_MSC426\%20Workshop06/term\%20papers\%2006/ Ling_06.pdf. Accessed 12 March 2014

Maezawa K, Kasahara K, Mori M (2010) A Traveling Wave Amplifier Based on Composite Right/Left Handed (CRLH) Transmission Lines Periodically Loaded with Resonant Tunneling Diode. In 2010 International Conference on Indium Phosphide \& Related Materials (IPRM), IEEE, Kagawa pp 1-4. doi:10.1109/ICIPRM.2010.5516365

Reddy M (1997) Schottky-collector resonant tunnel diodes for sub-millimeter-wave applications. Dissertation, University of California Santa Barbara

Slight TJ, Romeira B, Wang L, Figueiredo JML, Wasige E, Ironside CN (2008) A Liénard oscillator resonant tunnelling diodelaser diode hybrid integrated circuit: model and experiment. IEEE J Quantum Electron 44:1158-1163

\section{Submit your manuscript to a SpringerOpen ${ }^{\circ}$ journal and benefit from:}

- Convenient online submission

- Rigorous peer review

- Immediate publication on acceptance

- Open access: articles freely available online

- High visibility within the field

- Retaining the copyright to your article

Submit your next manuscript at $\mathbf{s p r i n g e r o p e n . c o m ~}$ 